\section{CD38 as a therapeutic target for adult acute myeloid leukemia and T-cell acute lymphoblastic leukemia}

Acute leukemia is a clonal expansion of malignant hematopoietic cells leading to impaired production of normal blood cells in the bone marrow (BM). Acute leukemia can be classified, according to the lineage involved, into acute myeloid leukemia (AML) or acute lymphoblastic leukemia (ALL). ${ }^{1} \mathrm{AML}$ is the most common form of acute leukemia in adults, accounting for over $80 \%$ of all diagnosed acute leukemias. Despite advances in the understanding of disease pathogenesis, the standard treatment protocols for adult acute leukemia have remained largely unchanged for the past decades. As a result, the overall 5-year survival in AML patients over 65 years of age is less than $5 \%,{ }^{2}$ and, although the initial responses are better in ALL, the overall outcome of relapsed ALL in adults is poor, with an overall 5-year survival of less than $7 \% .^{3}$ Hence, more and novel treatment options are warranted for both types of adult acute leukemia. Targeted immunotherapies, including monoclonal or bispecific antibodies and chimeric antigen receptor (CAR) T cells, are currently being developed and clinically tested in a wide variety of hematologic malignancies. CD38 is a transmembrane glycoprotein that can function as an adhesion partner for CD31 or as multifunctional ectoenzyme involved in the catabolism of $\mathrm{NAD}^{+}$and NADP. In the hematopoietic system, CD38 is expressed by lymphoid and myeloid cells, and is also expressed on red blood cells and platelets, with the highest expression found on plasma cells. This uniform, high expression on healthy and malignant plasma cells makes CD38 an ideal target for targeted immunotherapy in multiple myeloma (MM). Indeed, CD38 monoclonal antibodies (daratumumab, isatuximab, and MOR202) have been shown to be effective in relapsed or refractory MM patients and their use is gradually moving towards frontline therapy. ${ }^{4}$

The unmet need to develop novel, less toxic, but effective therapeutic strategies for adult acute leukemia, together with the safety and effectiveness of daratumum$\mathrm{ab}$ in MM, led us to evaluate the use of CD38 as a potential target in adult AML and T-cell ALL, and to investigate the efficacy of daratumumab against these diseases.

We first used flow cytometry to measure CD38 expression on leukemic blasts of 37 AML and 12 T-ALL patient samples taken at diagnosis. As shown, we observed a heterogeneous expression of CD38 in AML, while in T-ALL the expression of CD38 was more uniform (Figure $1 \mathrm{~A}$ and $\mathrm{B})$. CD38 expression did not correlate with French-American-British (FAB) classification nor European LeukemiaNet (ELN) risk stratification in AML or maturation status in T-ALL (data not shown). Moreover, there was no significant difference in CD38 expression between AML blasts at diagnosis and after chemotherapy in minimal residual disease positive $\left(\mathrm{MRD}^{+}\right) \mathrm{AML}$ patients (Figure 1C). Furthermore, no difference was seen between leukemic cells isolated either from peripheral blood or BM (Figure 1A and B; red vs. blue dots). Since we have recently shown that the level of CD38 expression observed on $\mathrm{CD}^{2} 8^{+}$regulatory $\mathrm{T}$ cells (Tregs) is sufficient for these cells to be affected by daratumumab treatment, we also analyzed the expression of CD38 on healthy
A

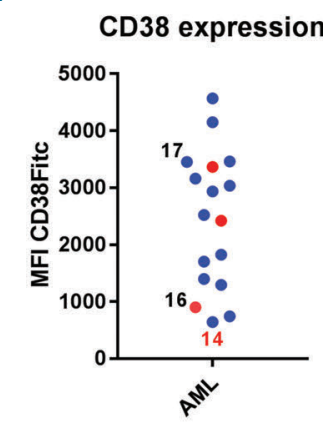

B

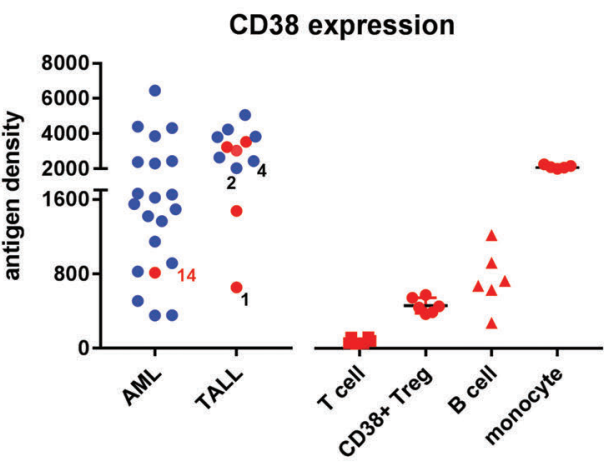

C

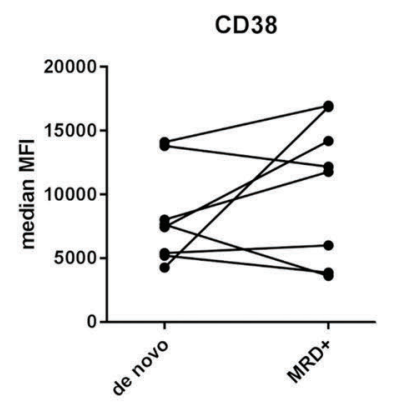

D

\begin{tabular}{|c|c|c|}
\hline Sample \# & risk group / subtype & cyto(genetics) \\
\hline AML16 & intermediate & NK (FIt3ITD, NPM1 mutated) \\
\hline AML17 & good & inv(16) \\
\hline T-ALL1 & immature single positive & complex \\
\hline T-ALL2 & immature single positive & NK \\
\hline T-ALL4 & immature MLL rearranged & $46, \mathrm{XY}, \mathrm{add}(1) \mathrm{p} 36, \mathrm{t}(6 ; 11)$ \\
\hline
\end{tabular}

Figure 1. CD38 expression in adult acute myeloid leukemia (AML) or T-cell acute lymphoblastic leukemia (T-ALL). (A and B) Evaluation of CD38 expression by flow cytometry. (A) Mean fluorescent intensity (MFI) of the leukemic blasts of 17 AML samples incubated with a FITC-conjugated CD38 mAb. (B) CD38 antigen density on leukemic blasts of $21 \mathrm{AML}$ and $12 \mathrm{~T}$-ALL patient samples and the density on $\mathrm{CD}^{+}{ }^{\top}$ cells, CD38 regulatory T cells (Tregs), CD19 ${ }^{+} \mathrm{B}$ cells, and CD33 ${ }^{+}$ monocytes of 6 healthy individuals determined using QuantiBRITE beads and a PE-conjugated CD38 mAb. Each dot represents a patient sample. Patient sample 14 (red number) is overlapping between the different cohorts shown in (A) and (B). The cells were isolated either from bone marrow (indicated by blue dots) or from peripheral blood (indicated by red dots). Black numbers indicate samples used for patient-derived xenograft (PDX) evaluation. (C). CD38 expression lev-

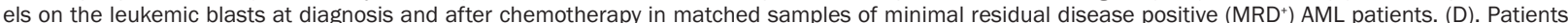
characteristics of samples selected for xenografting in huBMsc PDX model to test efficacy of daratumumab. NK: normal karyotype. 
peripheral blood mononuclear cells, including $\mathrm{CD}^{+} \mathrm{T}$ cells, $\mathrm{CD}_{38}{ }^{+}$Tregs, $\mathrm{CD} 19^{+} \mathrm{B}$ cells, and $\mathrm{CD} 33^{+}$monocytes. These data indicate that the CD38 expression found on the leukemic blasts of most samples in this study is higher than that found on CD38+ Tregs, and that, therefore, they could be targeted with daratumumab.

To test this, we selected 5 samples (2 AML and 3 T-ALL) (Figure 1D), representative of the wide spectrum of observed CD38 expression (Figure 1A), for evaluation in our in vivo human BM-like scaffold (huBMsc)-patientderived xenograft (PDX) model, which has been shown to closely recapitulate the human bone microenvironment and preserve the primary malignant phenotype. ${ }^{6,7}$ To this end, mice were subcutaneously implanted with four hybrid calcium phosphate scaffolds coated with human BM mesenchymal stromal cells that further develop into a human bone environment. Six weeks after implantation, mice were inoculated intrascaffold with leukemic cells of AML16 or AML17 or intravenously with T-ALL1 or T-ALL4 (Figure 2A). Three weeks after inoculation, the mice were treated with daratumumab 16 $\mathrm{mg} / \mathrm{kg}$ body weight or vehicle control intraperitoneally once a week for five weeks (Figure 2A). Eight weeks after the last injection, mice were sacrificed and the efficacy of daratumumab was evaluated by flow cytometric determination of the percentage of human CD45 positive cells in the scaffolds. Interestingly, daratumumab significantly reduced the tumor burden in all four PDX models with- out any evidence of toxicity (Figure 2B). Moreover, among 4 samples, only AML16 did not exclusively grow in the huBMsc but also engrafted in the murine BM, where a significant difference in tumor burden between daratumumab- and vehicle-treated mice was observed (data not shown). We next studied the effect of daratumumab in a PDX model, representing T-ALLs with more aggressive growth (T-ALL2) (Figure 3 ). The PDX model TALL2, besides the huBMsc, also colonized murine hematopoietic tissues (i.e. spleen and BM) upon intravenous injection, and when compared to the other T-ALL models, reached the humane end point in only 35 days instead of 15 weeks, thus showing its aggressive nature. In order to follow and visualize the engraftment and the therapeutic activity of daratumumab in real time, we gene-marked the leukemic cells of sample T-ALL2 with luciferase. Subsequently, these marked cells were inoculated intravenously into huBMsc-mice and treatment was initiated seven days after tumor cell injection. Leukemic cell growth was observed in the human environment in scaffolds as well as in mouse tissues (BM and spleen). Mice were treated with daratumumab $16 \mathrm{mg} / \mathrm{kg}$ body weight or vehicle control twice a week for four weeks by intraperitoneal administration (Figure 3A). Weekly bioluminescent imaging showed that mice receiving daratumumab had a significantly lower tumor burden as compared to vehicle-treated mice (Figure $3 B$ and C). Since the T-ALL cells showed splenic enlargement, we used splenic
A

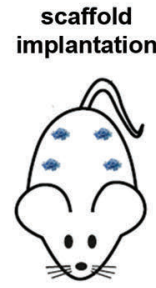

B

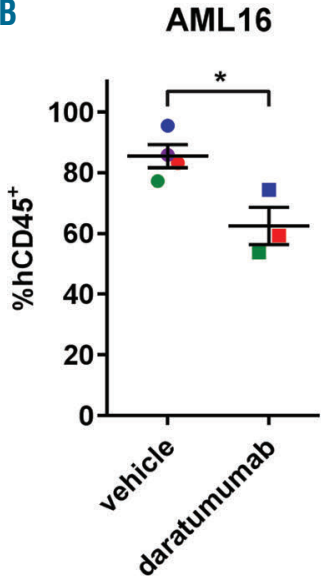

$\mathrm{TBI}=1.5 \mathrm{~Gy}$

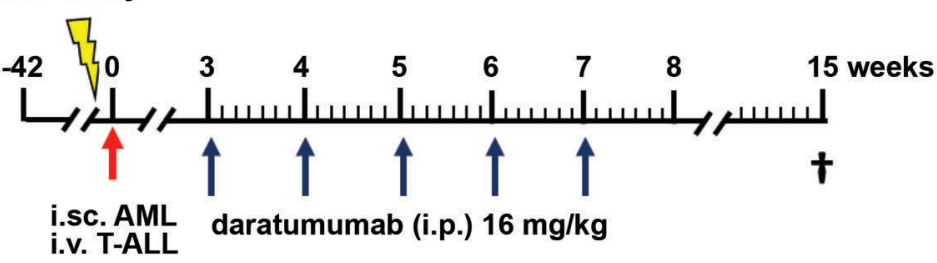

AML17

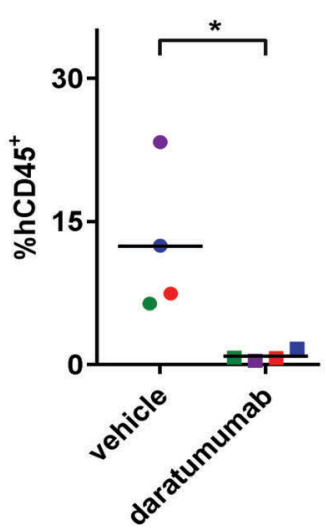

T-ALL 1

T-ALL 4

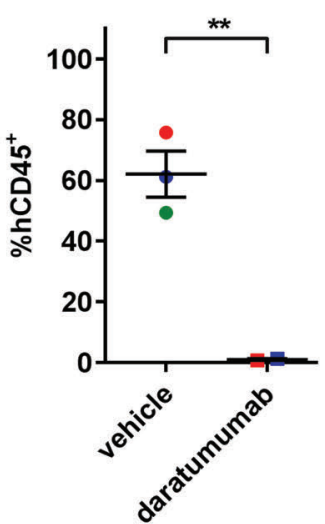

Figure 2. Daratumumab significantly reduces tumor burden in acute myeloid leukemia (AML) or T-cell acute lymphoblastic leukemia (T-ALL) patient derived xenograft (PDX) mice models. (A) Rag $2 / \gamma_{c}{ }^{-/}$mice were implanted with scaffolds coated with human mesenchymal stromal cells. Primary AML and T-ALL cells were injected into the scaffolds (i.sc.) or intravenously (i.v.), respectively. Mice were treated with vehicle control or daratumumab at the indicated time points (blue arrows) for five weeks. At week 15 mice were sacrificed and the tumor burden was analyzed by flow cytometry. (B). Percentage of CD45-positive (CD45+) cells in AML16, AML17, T-ALL1 and T-ALL4 PDX mice are plotted for vehicle and daratumumab treatment. Each dot represents the average of four scaffolds/mouse and the number of dots represents the number of mice per treatment group. $* P<0.05, * * P<0.01, * * * P<0.005$ compared to vehicle, using an unpaired $t$-test. TBI: total body irradiation. i.p.: intraperitoneal. 
A

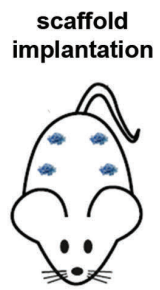

B

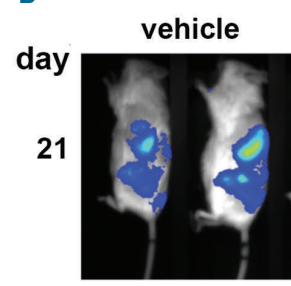

28
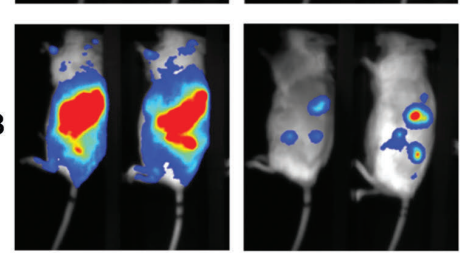

D

vehicle

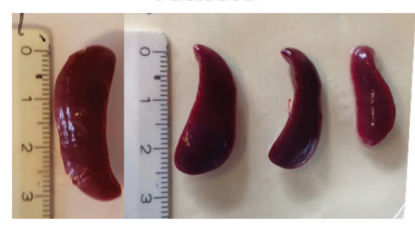

daratumumab

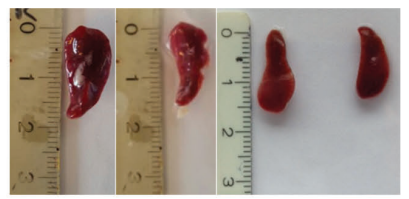

$\mathrm{TBI}=1.5 \mathrm{~Gy}$

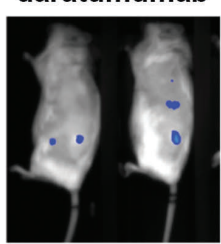

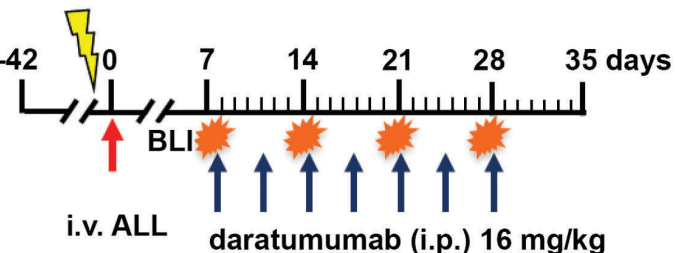

C

T-ALL 2 GFPLuc

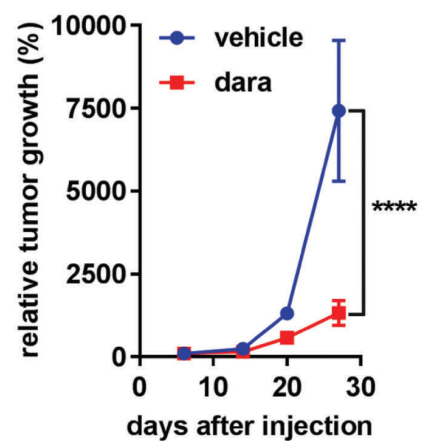

E

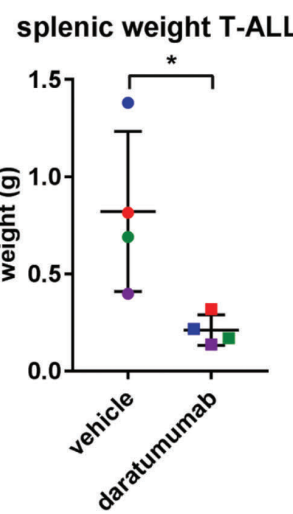

Figure 3. Daratumumab (dara) shows efficacy in a patient-derived xenograft (PDX) mouse model for aggressive Tcell acute lymphoblastic leukemia (TALL) growth. (A) Rag2 $\gamma_{c}{ }^{-1 /}$ mice carrying scaffolds coated with human mesenchymal stromal cells were inoculated (red arrow) with luciferase-transduced primary acute lymphoblastic leukemia (ALL) cells of T-ALL patient 2 (T-ALL2). Mice were treated with vehicle control or dara at the indicated time points (blue arrows). Mice were monitored weekly by bioluminescent imaging (BLI), and sacrificed at day 33 at which time point splenic weight was determined. (B). BLI at day 21 and 28 of dara treatment. Representative images are shown. (C). Tumor growth was determined by average photon emission intensity measured for a region of interest in time. The results are expressed as relative growth with the intensity at day 6 set to 100 . The lines represent the mean signal of 4 mice \pm Standard Error of mean. $* * * * P<0.001$ compared to vehicle, two-way ANOVA, Sidak's multiple comparison test. (D). Splenic weight was plotted for vehicle and dara-treated mice where the number of dots represents the number of mice per treatment group. ${ }^{*} P<0.05$ vehicle, MannWhitney test. TBI: total body irradiation; i.v.: intravenous injection; i.p.: intraperitoneal; g: gramms. weight as an additional parameter. We observed that the reduction in luciferase signals in bioluminescent imaging in the daratumumab-treated group coincided with an impressive reduction in splenic weight in the mice treated with daratumumab (Figure 3D and E). These observations indicate that daratumumab is very effective in both types of adult acute leukemia: AML and T-ALL.

In line with our findings that CD38 is a suitable therapeutic target for adult acute leukemia, Bride et al. recently showed the efficacy of daratumumab in pediatric TALL, particularly in early T-cell precursor (ETP)-ALL. ${ }^{8}$ In addition, Ichinohe's group, using CD38-targeting CAR T cells, showed effective elimination of HTLV-1 positive T-cell leukemia and AML.,10 Since it has been shown that CD38 expression was influenced by chemotherapy in BALL but not in T-ALL, ${ }^{8}$ we also analyzed the expression of CD38 on the leukemic blasts at diagnosis and after chemotherapy in $\mathrm{MRD}^{+} \mathrm{AML}$ patients and observed no significant difference (Figure 1C). We and others have shown that all-trans retinoic acid (ATRA) could enhance the lytic capacity of the CD38 monoclonal antibodies and CAR T cells by increasing the CD38 levels on the target cells. ${ }^{9-11}$ In addition, we have previously shown that EVI1-positive AML cases respond to ATRA by induction of differentiation, ${ }^{12}$ which coincides with the induction of CD38 expression (data not shown). Moreover, clinical and pre-clinical molecular studies show that patients with a NPM1 or IDH1 mutation, or an FLT3-ITD (which together constitute a sizable proportion of AML cases) could benefit from a combination of chemotherapy with ATRA. ${ }^{9,13}$ Hence, it would be interesting to evaluate combining daratumumab with ATRA as a therapeutic approach in adult AML and T-ALL. Finally, it has been demonstrated that the $\mathrm{CD} 34^{+} \mathrm{CD}_{38}{ }^{+}$population of certain AML samples contains all, or at least most, leukemia-initiating cells, ${ }^{14}$ again indicating the suitability of CD38 as a therapeutic target in AML. Taken together, our data show that daratumumab is effective in adult acute leukemia, and that targeting CD38 in these malignancies warrants further clinical evaluation. 
Jyoti Nair, ${ }^{1}$ Maria Themeli, ${ }^{1}$ Regina de Jong-Korlaar, ${ }^{1}$ Ruud W.J. Ruiter, ${ }^{1}$ Dino J Poddighe, ${ }^{2}$ Huipin Yuan, ${ }^{3}$ Joost D. de Bruin, ${ }^{3,4}$ Cert J. Ossenkoppele, Sonja Zweegman, Linda Sit, ${ }^{1}$ Tuna Mitis, ${ }^{1}$ Anton C.M. Martens, ${ }^{1}$ Noels W.C.J. van de Bonk ${ }^{1}$ and Richard W.J. Groen ${ }^{1}$

'Department of Hematology, Amsterdam UMC, VU University Medical Center, Cancer Center Amsterdam, the Netherlands; ${ }^{2}$ Department of Clinical Genetics, Amsterdam UMC, VU University Medical Center, Amsterdam, the Netherlands; ${ }^{3}$ Kuros Biosciences BV, Bilthoven, the Netherlands and ${ }^{4}$ The School of Engineering and Materials Science, Queen Mary University of London, UK

Acknowledgments: the authors would like to thank Dr. W. Noort for her technical and intellectual input.

Correspondence: RICHARD W.J. GROEN-r.groen@vumc.nl doi:10.3324/haematol.2018.192757

Information on authorship, contributions, and financial \& other discosure was provided by the authors and is available with the online version of this article at www. haematologica.org.

References

1. Blum S, Martins F, Lubbers M. Immunotherapy in adult acute leukemia. Leuk Res. 2017;60:63-73.

2. Their MS, Ershler WB, Jemal A, Yates JW, Beer MR. Outcome of older patients with acute myeloid leukemia: an analysis of SEER data over 3 decades. Cancer. 2013;119(15):2720-2727.

3. Marks DI, Rowntree C. Management of adults with T-cell lymphoblastic leukemia. Blood. 2017;129(9):1134-1142.

4. van de Dork N, Richardson PG, Malavasi F. CD38 antibodies in mut- tiple myeloma: back to the future. Blood. 2018;131(1):13-29.

5. Krejcik J, Casneuf T, Nijhof IS, et al. Daratumumab depletes CD38+ immune regulatory cells, promotes T-cell expansion, and skews Tcell repertoire in multiple myeloma. Blood. 2016;128(3):384-394.

6. Antonelli A, Noort WA, Jacques J, et al. Establishing human leukemia xenograft mouse models by implanting human bone marrow-like scaffold-based niches. Blood. 2016;128(25):2949-2959.

7. Groen RW, Mort WA, Raymakers RA, et al. Reconstructing the human hematopoietic niche in immunodeficient mice: opportunities for studying primary multiple myeloma. Blood. 2012;120(3):e9-e16.

8. Bride KL, Vincent TL, In SY, et al. Preclinical efficacy of daratumumab in T-cell acute lymphoblastic leukemia. Blood. 2018;131(9):995999.

9. Mihara K, Yoshida T, Ishida S, et al. All-trans retinoic acid and interferon-alpha increase CD38 expression on adult T-cell leukemia cells and sensitize them to $T$ cells bearing anti-CD38 chimeric antigen receptors. Blood Cancer J. 2016;6:e421.

10. Yoshida T, Mihara K, Take Y, et al. All-trans retinoic acid enhances cytotoxic effect of $\mathrm{T}$ cells with an anti-CD38 chimeric antigen receptor in acute myeloid leukemia. Chin Transl Immunology. 2016;5(12):e116.

11. Nijhof IS, Groen RW, Lokhorst HM, et al. Upregulation of CD38 expression on multiple myeloma cells by all-trans retinoic acid improves the efficacy of daratumumab. Leukemia. 2015;29(10):20392049.

12. Verhagen HJ, Sit MA, Rutten A, et al. Primary acute myeloid leukemia cells with overexpression of EVI-1 are sensitive to all-trans retinoic acid. Blood. 2016;127(4):458-463.

13. van Gild N, Verhagen H, Smith L. Reprogramming acute myeloid leukemia into sensitivity for retinoic-acid-driven differentiation. Exp Hematol. 2017;52:12-23.

14. Taussig DC, Miraki-Moud F, Anjos-Afonso F, et al. Anti-CD38 antibody-mediated clearance of human repopulating cells masks the hoterogeneity of leukemia-initiating cells. Blood. 2008;112(3):568-575.

haematological 2019; 104:e103 